\title{
Creating ultra-compact binaries in globular clusters through stable mass transfer
}

\author{
M. V. van der Sluys, F. Verbunt, and O. R. Pols
}

\author{
Astronomical Institute, Princetonplein 5, 3584 CC Utrecht, The Netherlands \\ e-mail: [sluys;verbunt;pols]@astro.uu.nl
}

Received 2 August 2004 / Accepted 23 October 2004

\begin{abstract}
A binary in which a slightly evolved star starts mass transfer to a neutron star can evolve towards ultra-short orbital periods under the influence of magnetic braking. This is called magnetic capture. We investigate in detail for which initial orbital periods and initial donor masses binaries evolve to periods less than 30-40 min within the Hubble time. We show that only small ranges of initial periods and masses lead to ultra-short periods, and that for those only a small time interval is spent at ultra-short periods. Consequently, only a very small fraction of any population of X-ray binaries is expected to be observed at ultra-short period at any time. If 2 to 6 of the 13 bright X-ray sources in globular clusters have an ultra-short period, as suggested by recent observations, their formation cannot be explained by the magnetic capture model.
\end{abstract}

Key words. stars: binaries: close - stars: evolution - Galaxy: globular clusters: general - X-rays: binaries

\section{Introduction}

The globular clusters belonging to our Galaxy house thirteen bright $\left(L_{\mathrm{X}} \gtrsim 10^{35} \mathrm{erg} \mathrm{s}^{-1}\right.$ in the $0.5-2.5 \mathrm{keV}$ range) $\mathrm{X}$-ray sources, neutron stars accreting from a low-mass companion. A surprisingly large fraction of these has ultra-short orbital periods of less than about $40 \mathrm{~min}$, as first noticed by Deutsch et al. (1996). Two of the five orbital periods known are $11.4 \mathrm{~min}$ and $20.6 \mathrm{~min}$ (or its alias $13.2 \mathrm{~min}$ ) for the sources in NGC 6624 and NGC 6712, respectively (Stella et al. 1987; Homer et al. 1996). The orbital periods of eight systems are not known, but for four of them indirect evidence points to an ultra-short period. This evidence consists of the absolute magnitude of the optical counterpart (van Paradijs \& McClintock 1994), of the energy distribution of the X-ray spectrum (Sidoli et al. 2001), and of the maximum flux reached during X-ray bursts (Kuulkers et al. 2003). Collating this evidence, Verbunt \& Lewin (2004, their Table 1) suggest that two more sources probably, and two others possibly have ultra-short orbital periods (in NGC 1851 and NGC 6652, and in NGC 7078 and Terzan 5, respectively). The 43.6 min period found by Deutsch et al. (1996) is not the period of the bright X-ray source in NGC 6652, but of a fainter source (Heinke et al. 2001).

Thus both among the known periods and among the suggested periods, about half of the bright X-ray sources have ultra-short orbital periods. This is in marked contrast to the period distribution of bright $\mathrm{X}$-ray sources in the galactic disk, where only one period much shorter than 40 min has been suggested so far (Wang \& Chakrabarty 2004).

Ultra-short-period binaries with a neutron star can be formed in a number of ways. An expanding giant star can engulf the neutron star, which then spirals in to form a binary with the helium-burning core. If mass transfer starts immediately after spiral-in, the donor is a helium-burning star (Savonije et al. 1986), if mass transfer starts only after a long time, the donor has evolved into a $\mathrm{CO}$ white dwarf or a $\mathrm{CO}$ white dwarf with helium mantle (Yungelson et al. 2002). The process requires a giant of higher mass than exists in globular clusters today; but the waiting time between end of the spiralin and onset of the mass transfer allows us to observe the mass transfer stage today of systems formed long ago. Indeed, it has been argued that this in fact is the dominant formation process for ultra-short-period binaries in globular clusters (Davies \& Hansen 1998; Rasio et al. 2000). Alternatively, it has been suggested that in a cluster, a neutron star can also in a collision with a giant expell its envelope and form a binary with its core (Verbunt 1987). It is not obvious that this leads to a binary sufficiently close to start mass transfer within the Hubble time (Rasio \& Shapiro 1991). A white dwarf donor implies an expanding orbit, and thus predicts an increasing orbital period.

Yet another scenario starts from a binary of a neutron star and a main-sequence star. The evolution of this binary depends critically on the initial orbital period. When the period is short, mass transfer is driven by loss of angular momentum, and the orbital period decreases with the donor mass until a minimum period is reached near 70 min (Paczynski \& Sienkiewicz 1981). We will call this a converging system. At the minimum period, the donor becomes degenerate, and further mass transfer expands the orbit. When the orbital period is long, mass transfer is driven by expansion of the donor star, and the orbit expands with the donor radius until the donor has transferred 
its full envelope (Webbink et al. 1983). These are diverging systems. However, for a narrow range of periods loss of angular momentum can still shrink the orbit for a slightly evolved donor. Due to its higher helium content, the donor becomes degenerate at smaller radius, and correspondingly shorter orbital period (Tutukov et al. 1985). Orbital periods shorter than 11 min can be reached (Podsiadlowski et al. 2002). These systems therefore converge, but the process may take more than a Hubble time. At $11.4 \mathrm{~min}$, the period derivative may be negative or positive, depending on whether the system is still on its way to the period minimum, or has already rebounded. We will refer to this scenario as magnetic capture.

The repeated observation that the $11.4 \mathrm{~min}$ period of the bright X-ray source in NGC 6624 is decreasing (van der Klis et al. 1993; Chou \& Grindlay 2001) would appear to indicate that the system evolved according to the magnetic capture scenario. However, it is not impossible that the negative period derivative is only apparent, the consequence of an acceleration of the binary in our direction, in the gravitational potential of the innermost part of the globular cluster. A more accurate position of the (optical counterpart to the) X-ray binary and a redetermination of the centre of the cluster shows that the X-ray source is much closer to the cluster centre than was thought before, and thus increases the probability that the measured period is affected by acceleration. Nonetheless, the measurement of a period decrease is a strong incentive to investigate the magnetic capture scenario in more detail.

A possible problem with the magnetic capture scenario is suggested by computations for binaries in the galactic disk, by Pylyser \& Savonije (1988). None of their calculated evolutions lead to periods of about 11 min within the Hubble time. Podsiadlowski et al. (2002) do not address this problem explicitly, but only show the time elapsed since the onset of mass transfer.

In this paper, we address the question under which circumstances the very short orbital periods observed in NGC 6624 and NGC 6712 are reached within the Hubble time, in the magnetic capture scenario described above. The parameters that we vary are the initial mass of the donor star, the initial orbital period (or more or less equivalently, the orbital period at which mass transfer starts), and the metallicity of the donor. In Sect. 2 we briefly describe the code that we use, and the algorithms specific to the evolutionary scenario that we study. In Sect. 3 we give the results for two specific cases, to compare with earlier work and to illustrate the possible evolution paths. We then describe the expected outcomes for an initial distribution of donor masses and initial orbital periods in Sect. 4. We find that orbital periods of 11.4 and $20.6 \mathrm{~min}$ are possible, but very unlikely in this scenario. The consequences of this conclusion are discussed in Sect. 5.

\section{Binary evolution code}

\subsection{The stellar evolution code}

We calculate our models using the STARS binary stellar evolution code, originally developed by Eggleton $(1971,1972)$ and with updated input physics as described in Pols et al. (1995).
Opacity tables are taken from OPAL (Iglesias et al. 1992), complemented with low-temperature opacities from Alexander \& Ferguson (1994).

The equations for stellar structure and composition are solved implicitly and simultaneously, along with an adaptive mesh-spacing equation. Convective mixing is modelled by a diffusion equation for each of the composition variables, and we assume a mixing length ratio $l / H_{\mathrm{p}}=2.0$. Convective overshooting is taken into account as in Schröder et al. (1997), with a free parameter $\delta_{\mathrm{ov}}=0.12$ calibrated against accurate stellar data from non-interacting binaries (Schröder et al. 1997; Pols et al. 1997). The helium core mass is defined as the mass coordinate where the hydrogen abundance becomes less than $10 \%$.

We use a version of the code (see Eggleton \& KiselevaEggleton (2002), hereafter EK02) that allows for nonconservative binary evolution, even though the evolution of only one component star is calculated in detail. The companion, in our case a neutron star, is treated as a point mass. With the adaptive mesh, mass loss by stellar winds or by Roche-lobe overflow (RLOF) in a binary is simply accounted for in the boundary condition for the mass. Spin-orbit interaction by tides is treated according to the equilibrium tide theory (Hut 1981) with a tidal friction timescale as given by EK02. This is taken into account by solving additional equations for the moment of inertia $I(r)$, the uniform stellar rotation frequency $\Omega_{\text {rot }}$, the orbital angular momentum $J_{\text {orb }}$ and the orbital eccentricity $e$. These equations (of which the latter three are independent of the interior structure and only depend on time) are also solved implicitly and simultaneously with the usual set of equations, at little extra computational cost. The rotation induces a centrifugal potential that influences the stellar structure through a reduction of the effective gravity. The centrifugal potential for each mesh point is averaged over a spherical shell. Rotationally induced mixing is not taken into account in this code.

Unlike EK02, we do not include their model for dynamodriven mass loss and magnetic braking. Rather we apply a magnetic braking law without accompanying mass loss, as discussed in Sect. 2.2. This facilitates direct comparison to previous binary evolution calculations in which similar assumptions have been made. Although we follow tidal interaction in detail, the effect on the current calculation is limited because the short orbital periods we consider ensure that the orbit is always circularised and synchronised with the stellar spin. However, exchange of angular momentum between spin and orbit is taken into account.

The initial hydrogen and helium abundances of our model stars are a function of the metallicity $Z: X=0.76-3.0 Z$ and $Y=0.24+2.0 Z$. In this research we use the metallicities $Z=$ 0.0001 (with $X=0.7597, Y=0.2402$ ), $Z=0.002$ (with $X=$ $0.754, Y=0.244$ ), $Z=0.01$ (with $X=0.73, Y=0.26$ ) and $Z=0.02$ (with $X=0.70, Y=0.28$ ).

\subsection{Angular momentum losses}

If the lower mass star in a binary fills its Roche lobe and starts to transfer mass to a more massive companion, the orbit will widen, unless there are enough angular momentum losses to 
compensate for this effect. We assume three sources of angular momentum loss from the system.

The most important source is magnetic braking. Due to magnetic braking, spin angular momentum is lost from the secondary and eventually, due to the tidal spin-orbit coupling, from the orbit. We use the formula given by Rappaport et al. (1983):

$$
\frac{\mathrm{d} J_{\mathrm{MB}}}{\mathrm{d} t}=-3.8 \times 10^{-30} M_{2} R^{4} \omega^{3} \text { dyn cm. }
$$

Like Podsiadlowski et al. (2002), we apply full magnetic braking when the mass of the convective envelope of the donor exceeds $2 \%$ of the total mass of the star, and if $q_{\text {conv }}<0.02$ reduce the strength of the magnetic braking in Eq. (1) by a factor of $\exp \left(1-0.02 / q_{\text {conv }}\right)$, where $q_{\text {conv }}$ is the mass fraction of the convective envelope of the star. The fact that the magnetic braking removes angular momentum from the spin of the star rather than directly from the orbit is different from Podsiadlowski et al. (2002). The main difference is that our study takes into account stellar spin at all, which influences the radius of the star and thus the moment at which Roche-lobe overflow commences.

For short orbital periods, gravitational radiation is a strong source of angular momentum loss. We use the standard description

$\frac{\mathrm{d} J_{\mathrm{GR}}}{\mathrm{d} t}=-\frac{32}{5} \frac{G^{7 / 2}}{c^{5}} \frac{M_{1}^{2} M_{2}^{2}\left(M_{1}+M_{2}\right)^{1 / 2}}{a^{7 / 2}}$

(Peters 1984).

The third way of angular momentum loss from the system is by non-conservative mass transfer. We assume that only a fraction $\beta$ of the transferred mass is accreted by the neutron star. The remainder is lost from the system, carrying away a fraction $\alpha$ of the specific angular momentum of the neutron star

$\frac{\mathrm{d} J_{\mathrm{ML}}}{\mathrm{d} t}=-\alpha(1-\beta) a_{1}^{2} \omega \dot{M}_{2}$,

where $a_{1}$ is the orbital radius of the neutron star and $\omega$ is the orbital frequency.

To keep the models simple, we applied no regular stellar wind to our models, so that all mass loss from the system and angular momentum loss due to this result from the nonconservative mass transfer described above.

\section{Binary models}

\subsection{Calculated grid}

Using the binary evolution code described in Sect. 2, we calculated an initial grid of models for $Z=0.01$, the metallicity of NGC 6624, and $Y=0.26$. We choose initial masses between 0.7 and $1.5 M_{\odot}$ with steps of $0.1 M_{\odot}$, and initial periods between 0.50 and 2.75 days, with steps of 0.25 days. Around the bifurcation period between converging and diverging systems, where the shortest orbital periods occur, we narrow the steps in $\mathrm{P}$ to 0.05 days.

We specify the bifurcation period more precisely as the longest initial period that leads to an ultra-short period, within

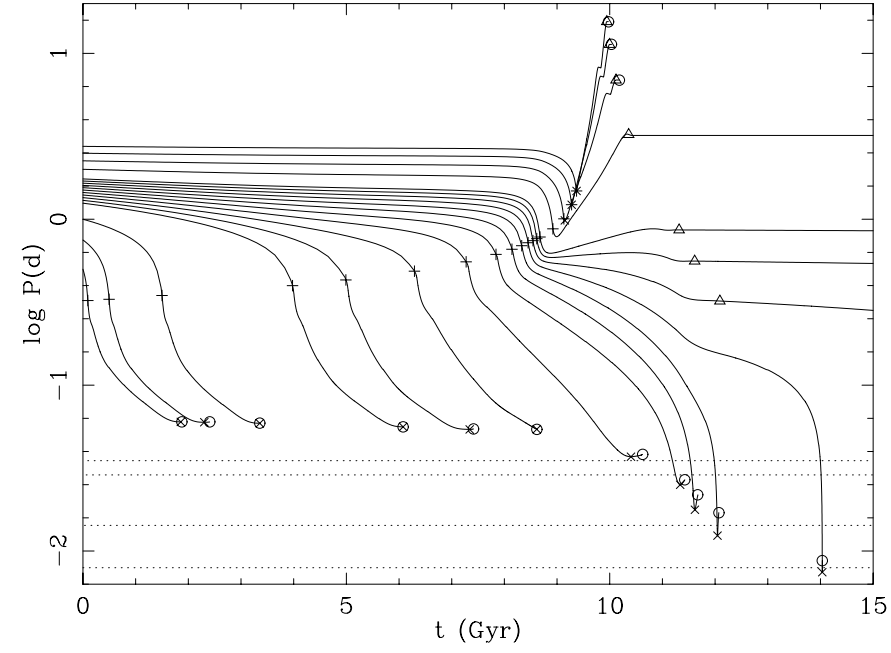

Fig. 1. Evolution of the orbital periods of selected systems with $Z=$ 0.01 , an initial secondary mass of $1.0 M_{\odot}$ and initial periods of 0.50 , $0.75,1.00,1.25,1.30,1.35,1.40,1.45,1.50,1.55,1.60,1.65,1.70$, $1.75,2.0,2.25,2.5$ and 2.75 days. The symbols mark special points in the evolution: + marks the start of Roche-lobe overflow (RLOF), $\times$ the minimum period, $\triangle$ the end of RLOF and $\bigcirc$ marks the end of the calculation. The four dotted horizontal lines show the orbital periods of the closest observed LMXBs in globular clusters: 11.4 and 20.6, and in the galactic disk: 41 and $50 \mathrm{~min}$.

a Hubble time. With this definition, the bifurcation period corresponds to the initial period of the binary that reaches its minimum period exactly after a Hubble time. This extra constraint is needed because there is no sharp transition between converging and diverging systems, especially since every diverging system will eventually converge due to gravitational radiation, if given the time. For instance, the system with an initial secondary mass of $1.1 M_{\odot}$ and an initial period of 0.90 days - that is shown to run out of the right of Fig. 2 at $\log P \approx-0.4-$ does converge to a period of slightly more than $5 \mathrm{~min}$, but only after almost $32 \mathrm{Gyr}$. This system is therefore considered to be diverging. Since the last part of the converging tracks in Figs. 1 and 2 is very steep, a system that reaches an ultra-short minimum period shortly after a Hubble time will usually have an orbital period at the Hubble time that is on the order of hours.

The total number of calculations for $Z=0.01$ is 150 ; 90 for the initial grid, and 60 for the finer grid. We follow Podsiadlowski et al. (2002) in choosing $\alpha=1$ and $\beta=0.5$ in Eq. (3). The orbital evolution of the systems with initial masses of 1.0 and $1.1 M_{\odot}$ is displayed in Figs. 1 and 2.

\subsection{Interpretation of the models}

Figure 1 shows that the models with the shortest initial periods converge to minimum periods of about $70 \mathrm{~min}$. After this, the stars become degenerate, and the orbits expand. Before the minimum period is reached, the stars become fully convective, thus mixing all of the star to a homogeneous composition. These stars have not yet formed a helium core, but are still a mixture of hydrogen and helium when they become degenerate. The stars with larger initial periods have a lower hydrogen abundance when they reach their minimum period. 


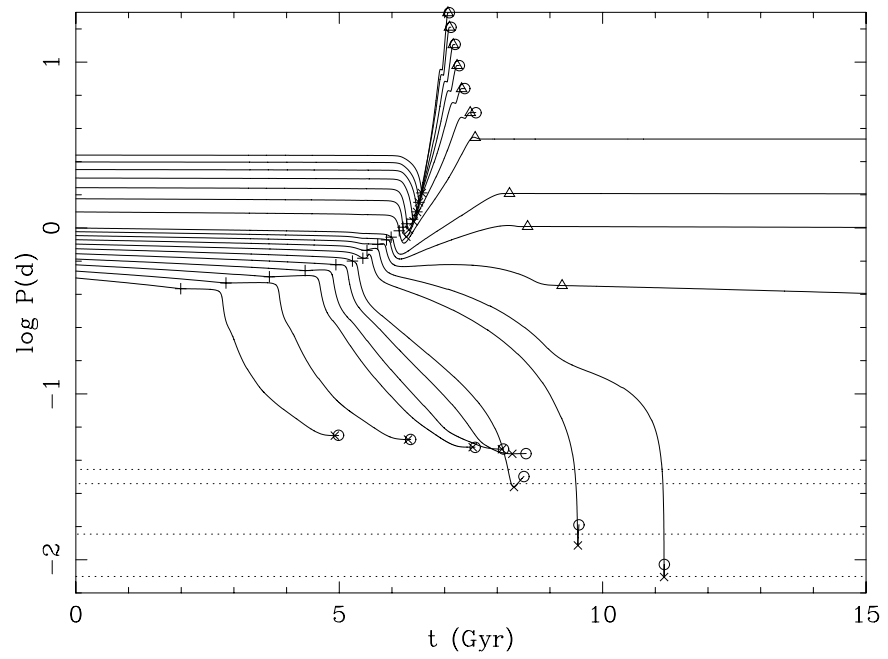

Fig. 2. Evolution of the orbital periods of selected systems with $Z=$ 0.01 , an initial secondary mass of $1.1 M_{\odot}$ and initial periods of 0.50 , $0.55,0.60,0.65,0.70,0.75,0.80,0.85,0.90,0.95,1.00,1.25,1.50$, $1.55,1.60,1.65,1.70,1.75,2.0,2.25,2.5$ and 2.75 days. See Fig. 1 for more details.

For the longest initial periods, the Roche lobe is filled in a later evolution stage and the evolutionary time scale is shorter, so that the star expands faster and the mass transfer rate is higher. Because of this, and the fact that the mass ratio is less than 1 , the angular momentum loss is not strong enough to shrink the orbit, so that it starts to expand shortly after mass transfer starts. These stars are sub-giants, and have a compact helium core inside their hydrogen envelopes. After they have transferred all of this envelope, they shrink and become helium white dwarfs. The systems with larger initial periods are more evolved when they fill their Roche lobes and produce more massive white dwarfs.

In between the smallest and largest initial periods, there are a number of models that reach orbital periods that are much shorter than $70 \mathrm{~min}$. This happens due to magnetic capture: the orbital period is reduced strongly under the influence of strong magnetic braking. When magnetic braking disappears, the orbit is close enough to shrink to ultra-short periods by angular momentum loss due to gravitational radiation. The magnetic captures come from models with a very narrow initial period range. The four models with $M_{\mathrm{i}}=1.0 M_{\odot}$ that reach a period less than $40 \mathrm{~min}$, for instance, have initial periods of $1.45,1.50$, 1.55 and 1.60 days, where the last model reaches the ultra-short period regime only after 14 Gyr. By interpolation, as described later in Sect. 4.1, we find that the models that reach a minimum period below $40 \mathrm{~min}$ and within $13.6 \mathrm{Gyr}$, have initial periods in the range 34.5-38.1 h. These stars fill their Roche lobes when their orbital periods are in the range of 14.3-17.2 h. The lowest orbital period reached, by the system with the initial period of $38.1 \mathrm{~h}$, is $12.0 \mathrm{~min}$, after $13.6 \mathrm{Gyr}$.

If one draws a vertical line in Fig. 1 at $11.5 \mathrm{Gyr}$ (about the age of the globular clusters), one can imagine that there is a distribution of observable X-ray binaries at that moment in time. The lowest orbital period found at that time is about $10^{-1.75}$ days, or $25 \mathrm{~min}$. All models with orbital periods higher

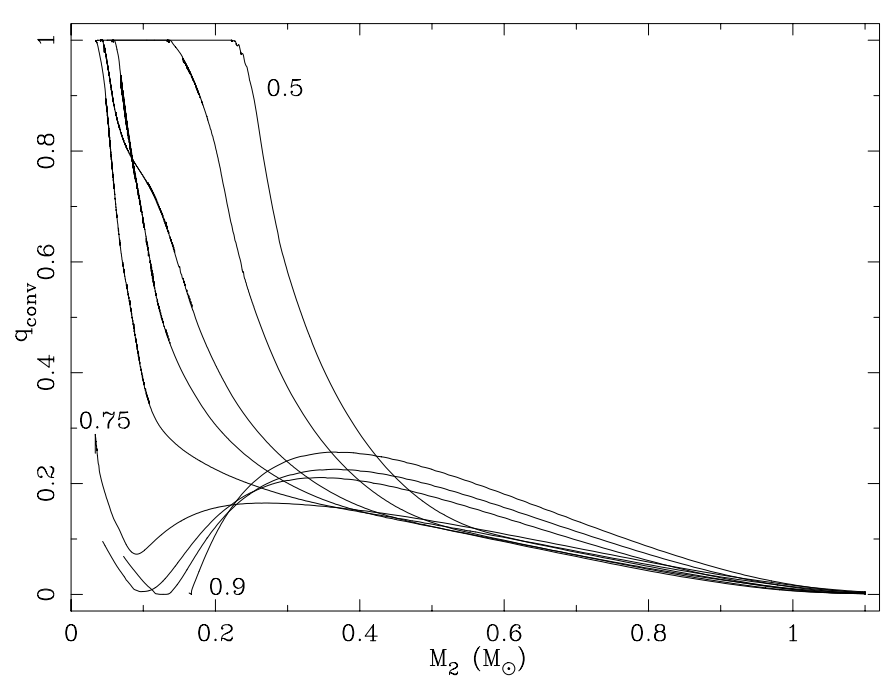

Fig. 3. Mass fraction of the convective envelope $\left(q_{\text {conv }}\right)$ as a function of the total mass of the donor, for the models with the shortest 9 initial orbital periods in Fig. 2. The numbers in the plot give the initial periods in days for that line. As evolution proceeds towards lower donor masses, the mass faction of the convective envelope increases. For the 5 models with initial periods between 0.5 and $0.7 \mathrm{~d}$, the total mass at which the star becomes fully convective is anti-correlated with the initial period. At initial periods of $0.75 \mathrm{~d}$ and longer, the initial increase of the mass fraction of the convective envelope is followed by a decrease.

than about 1 day have stopped mass transfer and will not be visible as X-ray binaries. Because the lines in Fig. 1 are steeper at lower periods, it is clear that the higher periods, around one day, will dominate.

Figure 2 shows the same data as Fig. 1, but for models with an initial secondary mass of $1.1 M_{\odot}$. The results are qualitatively similar, but the ultra-short period regime is reached from lower initial periods, and after a shorter period of time. We find that the models that reach periods lower than 40 min before 13.6 Gyr have initial periods of 18.0-20.9 h and fill their Roche lobes in the period range $15.1-18.2 \mathrm{~h}$. The system with the initial period of $18.0 \mathrm{~h}$ reaches $40 \mathrm{~min}$ after $8.3 \mathrm{Gyr}$, the system with a $20.9 \mathrm{~h}$ initial period has the smallest minimum period: $8.0 \mathrm{~min}$.

If we again imagine the period distribution at $11.5 \mathrm{Gyr}$, but now for Fig. 2, we see that the period range that we expect for mass transferring binaries is shifted downwards in period. Orbital periods as short as $10.6 \mathrm{~min}$ can now occur, and systems with periods over $9.5 \mathrm{~h}$ do not transfer mass anymore at that moment. With respect to the tracks in Fig. 1, we see that their density is much lower here. This is partially due to the fact that we use linear equally spaced periods at a lower initial period, so that they are more widely spaced in $\log P$.

Figure 3 illustrates the evolution of the convective envelope of a $1.1 M_{\odot}$ star for the grid models with initial periods between 0.5 and $0.9 \mathrm{~d}$. Looking at the models in the order of increasing initial period we find that in the first five the stars become fully convective at decreasing total masses. The first model that evolves towards ultra-short periods, with an initial period of $0.75 \mathrm{~d}$ is also the first model in which the donor never becomes fully convective: an initial increase of the mass 
Table 1. Properties for the donor stars of some of our grid models with $Z=0.01$ and $M_{\mathrm{i}}=1.1 M_{\odot}$ at their period minimum. The first three columns list the orbital period initially (at the ZAMS) in days, at Roche-lobe overflow $\left(P_{\text {rlof }}\right)$ in hours and the minimum period $\left(P_{\min }\right)$ in minutes. The next 11 columns show stellar properties at $P_{\min }$ : the age of the donor (since ZAMS), the logarithm of the mass transfer rate (expressed in $M_{\odot} \mathrm{yr}^{-1}$ ), the mass and luminosity of the donor, the logarithms of the effective temperature, the core temperature (both in K) and the central density (in $\mathrm{g} \mathrm{cm}^{-3}$ ), and the last four columns show the logarithms of the core and surface mass fractions of hydrogen and helium.

\begin{tabular}{|c|c|c|c|c|c|c|c|c|c|c|c|c|c|}
\hline$P_{\mathrm{i}}(\mathrm{d})$ & $P_{\text {rlof }}(\mathrm{h})$ & $P_{\min }(\mathrm{m})$ & $t$ (Gyr) & $\dot{M}_{\mathrm{tr}}$ & $M_{2}\left(M_{\odot}\right)$ & $\log L / L_{\odot}$ & $\log T_{\text {eff }}$ & $\log T_{\mathrm{c}}$ & $\log \rho_{\mathrm{c}}$ & $\log X_{\mathrm{c}}$ & $\log Y_{\mathrm{c}}$ & $\log X_{\mathrm{s}}$ & $\log Y_{\mathrm{s}}$ \\
\hline 0.50 & 10.3 & 80.7 & 4.92 & -10.31 & 0.060 & -3.64 & 3.33 & 6.44 & 2.53 & -0.23 & -0.40 & -0.23 & -0.40 \\
\hline 0.55 & 11.2 & 76.3 & 6.31 & -10.39 & 0.052 & -3.75 & 3.32 & 6.45 & 2.57 & -0.31 & -0.30 & -0.31 & -0.30 \\
\hline 0.60 & 12.2 & 68.7 & 7.53 & -10.49 & 0.042 & -3.85 & 3.32 & 6.54 & 2.81 & -1.46 & -0.02 & -0.53 & -0.16 \\
\hline 0.65 & 13.3 & 66.8 & 8.09 & -10.31 & 0.038 & -3.96 & 3.31 & 6.54 & 2.68 & -0.64 & -0.12 & -0.64 & -0.12 \\
\hline 0.70 & 14.4 & 62.7 & 8.28 & -10.36 & 0.043 & -3.67 & 3.38 & 6.72 & 2.82 & -1.32 & -0.03 & -0.89 & -0.07 \\
\hline 0.75 & 15.2 & 39.5 & 8.32 & -9.67 & 0.056 & -3.46 & 3.48 & 6.93 & 3.43 & -2.34 & -0.01 & -1.24 & -0.03 \\
\hline 0.80 & 15.8 & 17.6 & 9.53 & -8.53 & 0.074 & -3.97 & 3.45 & 7.01 & 4.08 & $-\infty$ & 0.00 & -1.51 & -0.02 \\
\hline 0.85 & 17.6 & 11.3 & 11.17 & -7.76 & 0.101 & -4.15 & 3.45 & 7.10 & 4.43 & $-\infty$ & 0.00 & -1.84 & -0.01 \\
\hline 0.90 & 19.1 & 5.1 & 31.85 & -6.62 & 0.164 & -4.89 & 3.34 & 6.81 & 5.05 & $-\infty$ & 0.00 & -1.10 & -0.04 \\
\hline
\end{tabular}

Table 2. Some properties for two of our grid models with $Z=0.01$ and $M_{\mathrm{i}}=1.1 M_{\odot}$ at selected orbital periods. First row: initial (ZAMS) parameters. Rows 2-6: the model with $P_{\mathrm{i}}=0.75 \mathrm{~d}$ and $P_{\min }=39.5$ min. Rows $7-11$ : the model with $P_{\mathrm{i}}=0.85 \mathrm{~d}$ and $P_{\min }=11.3$ min. $\dot{M}_{\text {tr }}$ in Col. 3 is expressed in $M_{\odot} \mathrm{yr}^{-1}$ and $T_{\text {eff }}$ in Col. 7 in Kelvin. The last five columns give the logarithm of the surface mass fractions of the elements described.

\begin{tabular}{llllllllllll}
\hline \hline$P_{\text {orb }}(\min )$ & $t(\mathrm{Gyr})$ & $\log \dot{M}_{\mathrm{tr}}$ & $\log -\dot{P}_{\text {orb }}$ & $M_{2}\left(M_{\odot}\right)$ & $\log L / L_{\odot}$ & $\log T_{\text {eff }}$ & $\log \mathrm{H}$ & $\log \mathrm{He}$ & $\log \mathrm{C}$ & $\log \mathrm{N}$ & $\log \mathrm{O}$ \\
\hline$P_{\text {ZAMS }}$ & 0.000 & - & - & 1.100 & 0.17 & 3.79 & -0.14 & -0.59 & -2.75 & -3.28 & -2.30 \\
& & & & & & & & & & & \\
80.0 & 8.023 & -10.11 & -12.56 & 0.097 & -2.44 & 3.59 & -0.46 & -0.19 & -5.12 & -2.50 & -2.36 \\
60.0 & 8.147 & -9.97 & -12.48 & 0.086 & -2.66 & 3.59 & -0.57 & -0.14 & -5.07 & -2.42 & -2.44 \\
50.0 & 8.205 & -9.81 & -12.49 & 0.079 & -2.82 & 3.58 & -0.70 & -0.10 & -5.01 & -2.35 & -2.53 \\
45.0 & 8.236 & -9.72 & -12.54 & 0.074 & -2.95 & 3.57 & -0.82 & -0.08 & -4.96 & -2.31 & -2.62 \\
39.5 & 8.317 & -9.67 & $-\infty$ & 0.056 & -3.46 & 3.48 & -1.24 & -0.03 & -4.90 & -2.23 & -2.88 \\
& & & & & & & & & & & \\
40.0 & 11.145 & -9.92 & -11.86 & 0.124 & -1.64 & 3.88 & -0.48 & -0.18 & -5.22 & -2.50 & -2.36 \\
30.0 & 11.156 & -9.51 & -11.68 & 0.122 & -1.87 & 3.86 & -0.51 & -0.17 & -5.23 & -2.49 & -2.36 \\
20.0 & 11.163 & -9.06 & -11.41 & 0.120 & -2.46 & 3.77 & -0.57 & -0.14 & -5.18 & -2.48 & -2.37 \\
15.0 & 11.165 & -8.53 & -11.26 & 0.117 & -3.21 & 3.63 & -0.68 & -0.11 & -5.11 & -2.47 & -2.39 \\
11.3 & 11.167 & -7.76 & $-\infty$ & 0.101 & -4.15 & 3.45 & -1.84 & -0.01 & -4.81 & -2.31 & -2.62 \\
\hline
\end{tabular}

fraction of the convective envelope is followed by a decrease. For initial periods of $0.85 \mathrm{~d}$ and $0.9 \mathrm{~d}$ the convective envelope disappears completely. The general trend with increasing initial period that is visible in Fig. 3, is the consequence of an increasing helium abundance in the core. The cores with a higher helium abundance tend to be hotter and thus more stable against convection. The absence of convection in the core in turn keeps the helium abundance high. The third model, with an initial period of $0.6 \mathrm{~d}$ shows a track that is slightly different from those of the neighbouring models. This model becomes almost fully convective, but the central $10^{-4} M_{\odot}$ does not, and as a consequence the mixing from the core to the surface is suppressed. We have repeated this calculation with a slightly different convective mixing efficiency and find the same results.

Table 1 lists some properties of the same nine models shown in Fig. 3 at their period minimum. The first five models all have minimum periods more than $1 \mathrm{~h}$ and more than $1 \%$ hydrogen in the core at their minimum, whereas the cores of the last four models consist for more than $99 \%$ of helium. With decreasing minimum period, the mass transfer rates increase rapidly and the luminosities of the donors decrease.
In Table 2 we list some observational properties along the evolutionary tracks of two of our grid models with $Z=0.01$ and $M_{\mathrm{i}}=1.1 M_{\odot}$.

Although we find that it is possible to reach orbital periods below 40 min without spiral-in, but due to magnetic capture instead, it seems that one has to select an initial period carefully in order to actually do so. We also find that it is possible to construct a model that has a minimum period as low as the observed $11.4 \mathrm{~min}$ in a time span smaller than the Hubble time. The question arises, however, what the chances are that such a system is indeed formed in a population of stars. In order to quantify this, we will expand our parameter space to the entire grid we calculated and do statistics on these tracks in Sect. 4.

\subsection{Bifurcation models}

For an initial secondary mass of $1.1 M_{\odot}$, the grid models with initial periods of 0.85 days and 0.90 days bracket the bifurcation period. Some timescales that can explain this difference are shown in Fig. 4. The evolution of both models is rather similar in the beginning, except for the small difference in 


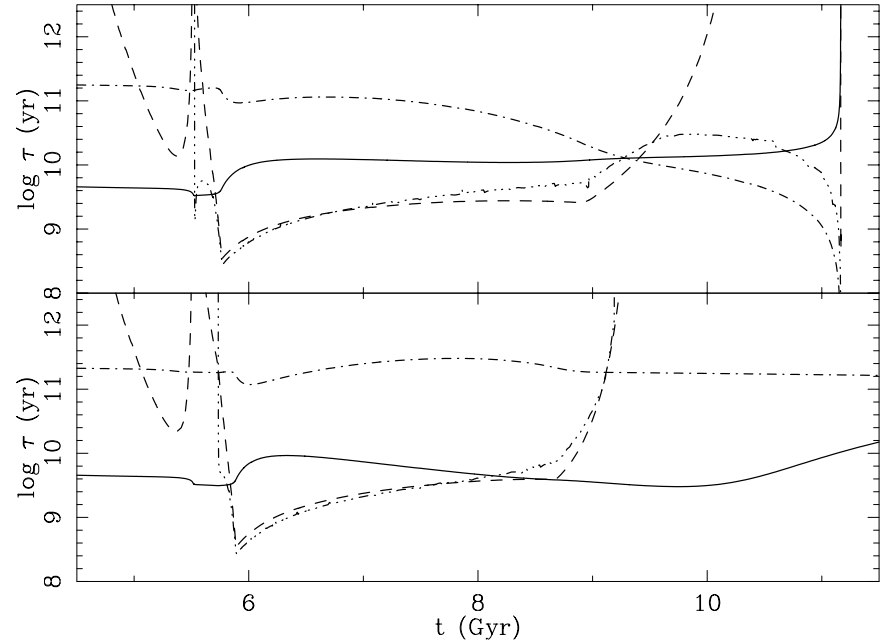

Fig. 4. Timescales of the models that bracket the bifurcation period for $1.1 M_{\odot}$. Upper panel a): model with $P_{\mathrm{i}}=0.85 \mathrm{~d}$. Lower panel b): model with $P_{\mathrm{i}}=0.90 \mathrm{~d}$. The line styles represent the different timescales: Solid line: nuclear evolution timescale $\left(M / M_{\odot}\right) /\left(L / L_{\odot}\right) \times$ $10^{10} \mathrm{yr}$, dashes: magnetic braking timescale $J_{\text {orb }} / \dot{J}_{\mathrm{MB}}$, dash-dot: gravitational radiation timescale $J_{\mathrm{orb}} / \dot{J}_{\mathrm{GR}}$, dash-dot-dot-dot: mass transfer timescale $M / \dot{M}_{\mathrm{tr}}$. See the text for a discussion.

orbital period, that stays about constant during the main sequence. The wider system has a larger Roche lobe and thus the donor fills its Roche lobe at a slightly later stage of its evolution. At this point, the evolutionary timescale of the donor is shorter than that in the closer system, and it can form a well defined helium core. When the envelope outside this core has been reduced by mass transfer to $\simeq 0.03 M_{\odot}$, it collapses onto the core, mass transfer stops, and magnetic braking disappears before the magnetic capture is complete. Gravitational radiation is then the only term of angular momentum loss and it is not strong enough to shrink the orbit to the ultra-short period regime within the Hubble time.

In the closer system, the evolutionary timescale of the donor is slightly larger and its helium core mass is slightly smaller. At approximately $9 \mathrm{Gyr}$ mass transfer has stripped the donor to such extend that hotter layers emerge at the surface, the convective envelope of the star becomes very thin and magnetic braking is strongly reduced (see the discussion with Fig. 3). Figure 4 shows that this happens at the moment where the gravitational radiation timescale becomes shorter than the evolutionary timescale of the donor, so that angular momentum loss remains sufficient to shrink the orbit from the hour to the minute regime.

\section{Statistics}

\subsection{Interpolation between models}

In order to do statistics on our models, we have to interpolate between the calculated models to get a time-period track, that gives the orbital period of a system as a function of time, for a given initial orbital period $P_{\mathrm{i}}$.

Before we can interpolate between two calculated tracks, we must first divide the tracks into similar parts of evolution.
We choose three parts: i) the part between ZAMS and the beginning of RLOF; ii) the part between the beginning of RLOF and the moment where the minimum period $\left(P_{\min }\right)$ was reached; and iii) the part between $P_{\min }$ and the end of the calculation. Each of these parts is redistributed into a fixed number of data points, equally spaced in the path length of that part and determined by a polynomial interpolation of the third degree. The path length is the integrated track in the $t-\log P$ plane, and defined as

$\ell=\sum_{i} \sqrt{\left(\frac{t(i)-t(i-1)}{\Delta t}\right)^{2}+\left(\frac{\log P(i)-\log P(i-1)}{\Delta \log P}\right)^{2}}$,

where $\Delta t=t_{\max }-t_{\min }$ and $\Delta \log P=\log P_{\max }-\log P_{\min }$. Thus, each part of all tracks contains the same number of points, and each point on these parts marks about the same moment in evolution in two different tracks.

Next, we interpolate between two tracks, to calculate the track for the given initial period. Because the tracks differ considerably between the shortest and longest initial period, we use linear interpolation between two adjacent tracks, that are always rather similar. Each track is thus interpolated point-bypoint between each pair of corresponding points from the two adjacent tracks, to get the time and the orbital period.

Once the interpolated track is known, we interpolate within the track, to obtain the orbital period at a given moment in time. For this, we use a polynomial interpolation of the fourth degree. For some models the second part of a track consists of one point, because the beginning of RLOF marks the minimum period. For interpolations involving this point, we use a third degree polynomial interpolation.

A handful of models crash after they have stopped mass transfer, for instance the models with the highest initial period in Figs. 1 and 2. These systems will not give observable X-ray sources, but some of these tracks may be needed for the interpolation. We continued the orbital evolution of the most important of these models analytically, under the influence of gravitational radiation only, until the orbit becomes so small that the star's Roche lobe touches its surface. We consider the orbital period at which mass transfer recommences as the minimum period. We assume a constant radius of the star since the last converged model, which probably means that we overestimate the minimum period a bit in these cases.

\subsection{Results for $Z=0.01$}

In Sect. 3, we have found that we can create LMXBs with periods down to $11 \mathrm{~min}$ or perhaps even less, within a Hubble time. We also saw, however, that one has to select the initial period carefully to create a model that reaches such a low period, and that the system spends very little time on this minimum period. In order to investigate how probable it is to observe ultracompact binaries, we select random points on random tracks like the ones in Figs. 1 and 2 and convert the result into a histogram. We perform this operation in the following way.

For a fixed initial secondary mass, we draw a random initial period, between 0.50 and 2.75 days, from a flat distribution in $\log P$. We then interpolate the time-period track that 

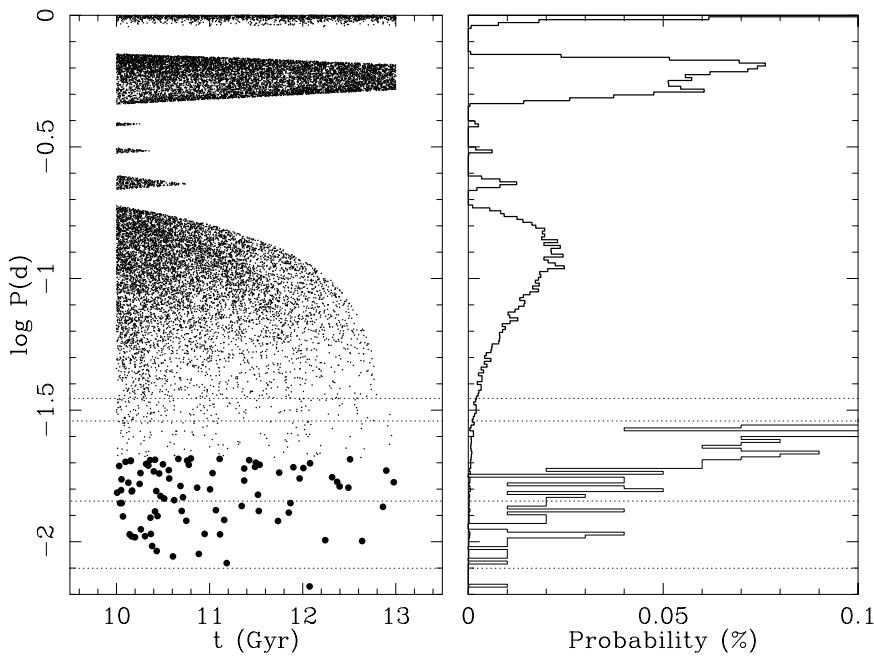

Fig. 5. Statistics results for the $1.1 M_{\odot}$ models. Left panel a): results from the draw of one million random initial periods and times. Each dot represents the orbital period of the selected system at the selected time. Only models that were converging and transferring mass at that time were accepted, about $10.5 \%$ of the total number. The peaks at the higher orbital periods are artefacts, caused by interpolation between models with and without mass transfer. Dots below $P=30 \mathrm{~min}$ are plotted larger for clarity. Right panel $\mathbf{b}$ ): a histogram displaying the fraction of systems found at a certain orbital period, at any time between 10 and 13 Gyr. The $\log P$-axis was chosen to be vertical, to correspond to the vertical axis in the left panel. The thick line displays the data corresponding to the horizontal axis, the thin line is the short-period tail of the same data, multiplied by a factor of 100 in the horizontal (probability) direction. The dotted horizontal lines are the orbital periods of the four observed LMXBs mentioned in Fig. 1.

corresponds to this initial period, using the method described in Sect. 4.1. For each point on this track, an estimate for the mass transfer rate is obtained by interpolating in the logarithm of the calculated mass transfer rates. For points without mass transfer, we adapt a value of $\dot{M}_{\mathrm{tr}}=10^{-35} M_{\odot} \mathrm{yr}^{-1}$, so that we can take its logarithm. This introduces some irregularities, like the peaks around $\log P(d)=-0.5$ in Fig. 5, where interpolation between models with and without mass transfer, and interpolation between converging and diverging models play a role. This is usually only the case at orbital periods of several hours or more, and hence it is of no consequence for the ultra-compact binaries.

Once the time-period track is calculated, we draw a random moment in time, from a linear distribution between 10 and 13 Gyr, the approximate ages of globular clusters, and interpolate within the track to obtain the orbital period at that random moment. We accept only systems that have not evolved beyond their minimum period, firstly because of the negative period derivative measured for the 11.4 min system in NGC 6624, and secondly because the evolution code we use can generally not calculate far beyond the period minimum. We also estimate the mass transfer rate at that moment, again by interpolating in $\log \dot{M}_{\mathrm{tr}}$. We reject all systems with a mass transfer rate $\dot{M}_{\text {tr }}<10^{-20} M_{\odot} \mathrm{yr}^{-1}$, because it is unlikely that they have any mass transfer at that moment and will therefore not be an X-ray source.

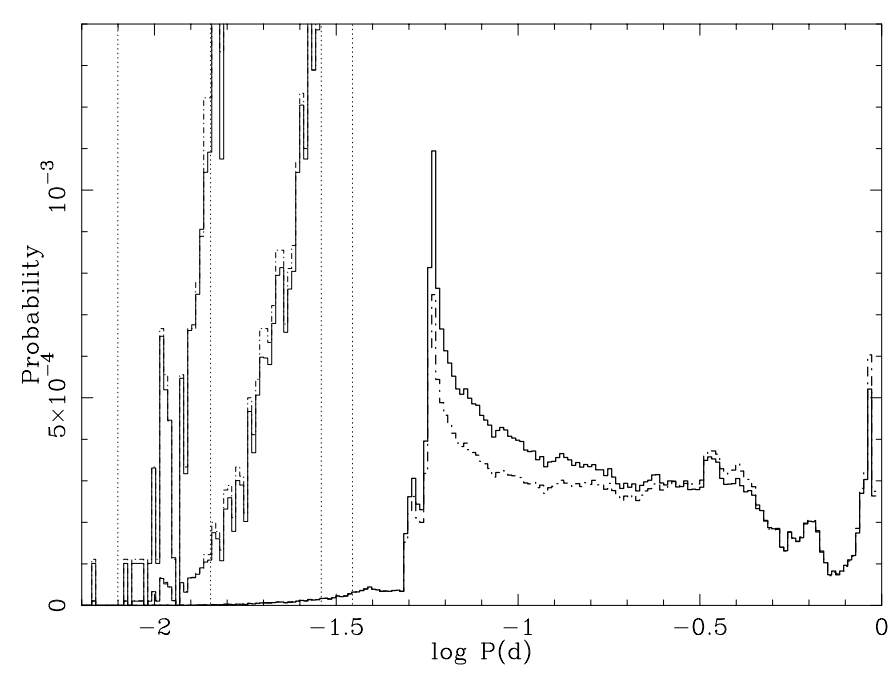

Fig. 6. Probability distribution for all models with $Z=0.01$. The solid line represents the sum of the distributions of the different masses weighed with the Salpeter birth function, the dash-dotted line assumes a flat distribution in mass. The thin lines below $\log P(d)=-1.3$ and below $\log P(d)=-1.7$ are the same data, multiplied with 100 and 1000 respectively. The four vertical, dotted lines show the orbital periods of the four observed LMXBs mentioned in Fig. 1.

If we repeat this procedure many times, we can create a histogram that displays the expected distribution of orbital periods of a population of converging LMXBs (with all the same initial secondary masses) after $10-13$ Gyr. The results for $1.1 M_{\odot}$ and $Z=0.01$ are shown in Fig. 5.

To simulate a population consisting of stars of different masses, one should interpolate between the tracks as we did for the period. The tracks are too different from each other to ensure correct results. It would require a large number of extra models to be able to interpolate between the masses correctly. Instead, we choose to add the period distributions of the different masses to simulate such a population. We do this for two different assumptions for the mass distribution: the Salpeter birth function, and a flat distribution. The results are shown in Fig. 6.

We see that there is little difference between the two weighing methods. This assures that although we do not know the initial distribution of the mass, it is of little influence on this result. Especially the short-period tails of the distributions are almost equal. In a sample of $10^{7}$ systems we find one converging system with a period of about $11 \mathrm{~min}$ and 15 systems with a period of $20 \mathrm{~min}$.

\subsection{Results for other metallicities}

The whole exercise we described in section Sect. 3.1, 4.1 and 4.2 is also applied to models for $Z=0.0001, Z=0.002$, and $Z=0.02$, in order to see the effect of metallicity on the expected distributions. For $Z=0.02$ we calculate the same initial grid as we did for $Z=0.01$, between $P_{\mathrm{i}}=0.5-2.75$ days for $M_{\mathrm{i}}=0.7-1.3 M_{\odot}$, but $P_{\mathrm{i}}=0.55-3.025$ days for $M_{\mathrm{i}}=1.4$ and $1.5 M_{\odot}$, since these stars even at the ZAMS do not fit in an orbit with $P=0.5$ days. For $Z=0.002$ we use the same 


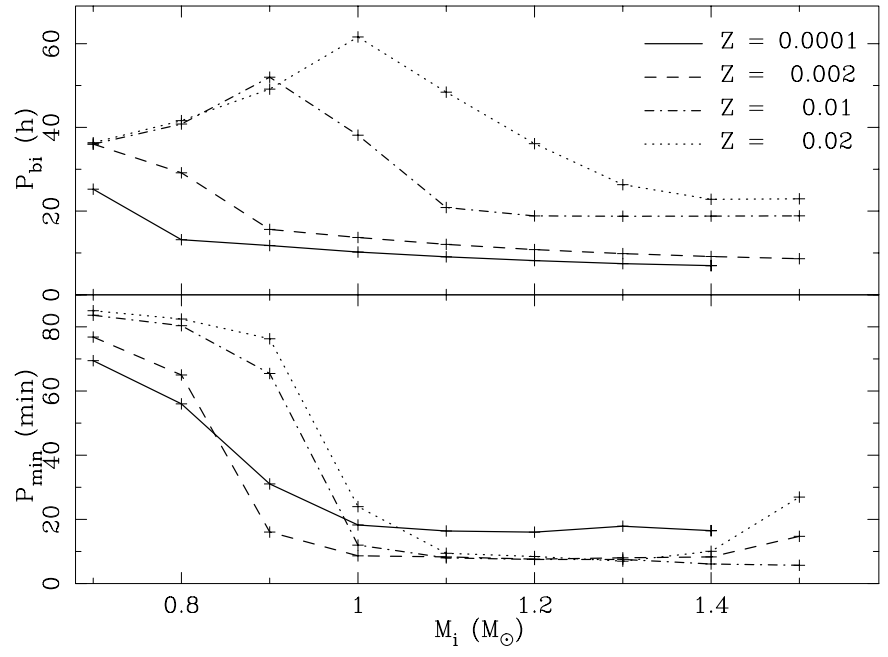

Fig. 7. Bifurcation periods and minimum periods as a function of the initial mass for the four metallicities. Upper panel a) the bifurcation period (in hours) between systems that converge and systems that do not converge within a Hubble time. Lower panel b) the minimum period (in minutes) that can be reached within a Hubble time as a function of the initial secondary mass. The different line styles display the different metallicities, as indicated in the upper panel. The data point for $Z=0.0001, M_{\mathrm{i}}=1.5 M_{\odot}$ is missing in both panels, because the bifurcation period for these systems is lower than the period at which such a donor fills its Roche lobe at ZAMS.

initial mass range, but it turns out that for $M_{\mathrm{i}}=1.0-1.5 M_{\odot}$ the bifurcation period lies very close to or lower than 0.5 days (see Fig. 7). We therefore shift the minimum initial period to 0.35 days for $M_{\mathrm{i}}=1.0,1.4$ and $1.5 M_{\odot}$, and to 0.4 days for $M_{\mathrm{i}}=1.1-1.3 M_{\odot}$. For $Z=0.0001$, the minimum initial period is shifted to $0.3 \mathrm{~d}$ for $0.7-1.2 M_{\odot}$ and even to $0.28 d$ for $1.3-1.5 M_{\odot}$. For $Z=0.0001$ and $M_{\mathrm{i}}=1.5 M_{\odot}$, the initial period at which a ZAMS star fills its Roche lobe is higher than the bifurcation period. Stars with higher $Z$ have larger radii and often do not fit in these tight orbits. We shift the upper limit for the period range from which we took random values accordingly, so that the size of the range (in $\log P$ ) did not change. Since the bifurcation period for the lower metallicity models lies lower, we also have to pinpoint better to calculate the interesting models around it. We therefore narrow the grid to steps of $0.01 \mathrm{~d}$ around the bifurcation period for $Z=0.002$ and $Z=0.0001$, and even down to $0.001 \mathrm{~d}$ for the last metallicity.

The bifurcation periods for the different masses are plotted in Fig. 7a. There is a trend in metallicity in the sense that the dotted line of $Z=0.02$ could be moved down and left to fall over that of $Z=0.01$ and further to reach that of $Z=0.002$ and $Z=0.0001$. Figure $7 \mathrm{~b}$ shows the minimum periods for the systems that have the bifurcation period for that mass as their initial period. The trend that is shown can be explained the fact that low mass stars with a lower metallicity reach the TAMS before the Hubble time and are therefore eligible for magnetic capture, whereas low mass stars of higher $Z$ do not.

The results of the statistics for $Z=0.0001, Z=0.002$ and $Z=0.02$ are plotted in Figs. 8-10 in the same way as the results for $Z=0.01$ in Fig. 6, so that they can easily be compared. All four distributions are also plotted in a cumulative plot in

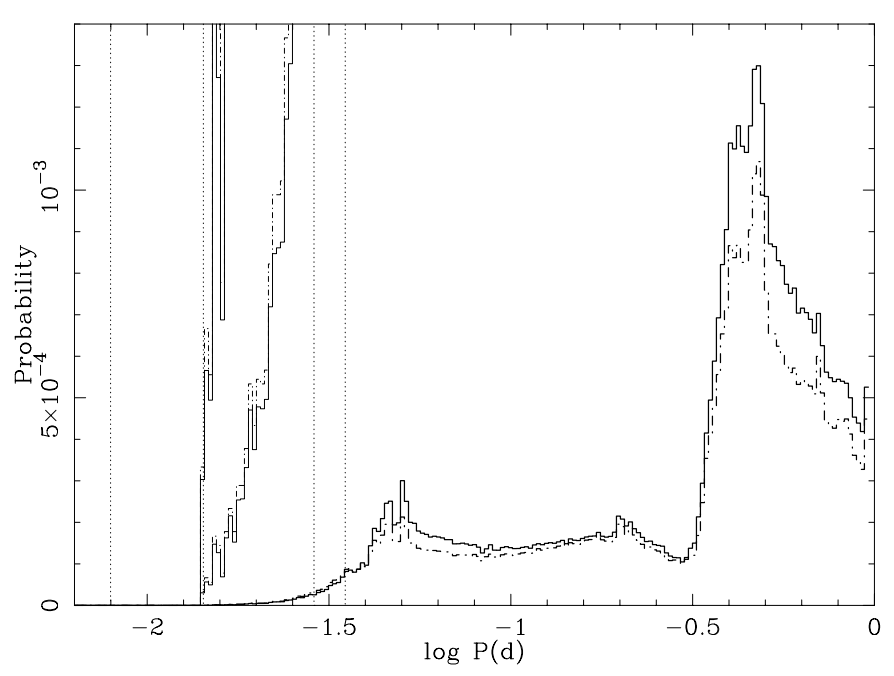

Fig. 8. Probability distribution of the orbital periods for all models with $Z=0.0001$. The characteristics of this plot are the same as in Fig. 6.

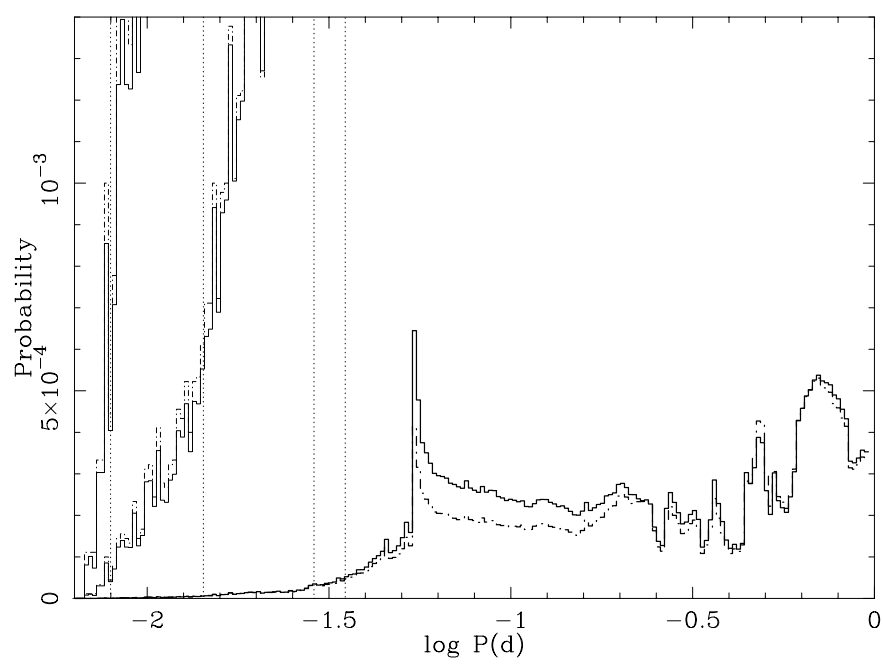

Fig. 9. Probability distribution of the orbital periods for all models with $Z=0.002$. The characteristics of this plot are the same as in Fig. 6.

Fig. 11, showing the fraction of systems with an orbital period below some value, so that they can be compared directly.

The most remarkable feature in the three distributions with the higher metallicities is the sharp drop of the number of predicted systems below $\log P(d)=-1.25$, or about $80 \mathrm{~min}$. This is due to the systems with low initial mass $\left(0.7-0.9 M_{\odot}\right)$, that reach their minimum periods there because they evolve too slow to reach ultra-short periods before the Hubble time, and remain relatively long at this period. Models with $Z=0.0001$ evolve more quickly, and although most models do not reach ultra-short periods, they are substantially lower than $80 \mathrm{~min}$ and can even reach $31 \mathrm{~min}$ in the case of $M_{\mathrm{i}}=0.9 M_{\odot}$. The drop is therefore less sharp for the lowest metallicity we used.

The lower mass stars dominate in roughly the $\log P$-range $-1.25--0.6$, as can be seen from the fact that here the solid line for a Salpeter weighted addition of the masses that favours low mass stars is higher than the dash-dotted line for a flat mass 


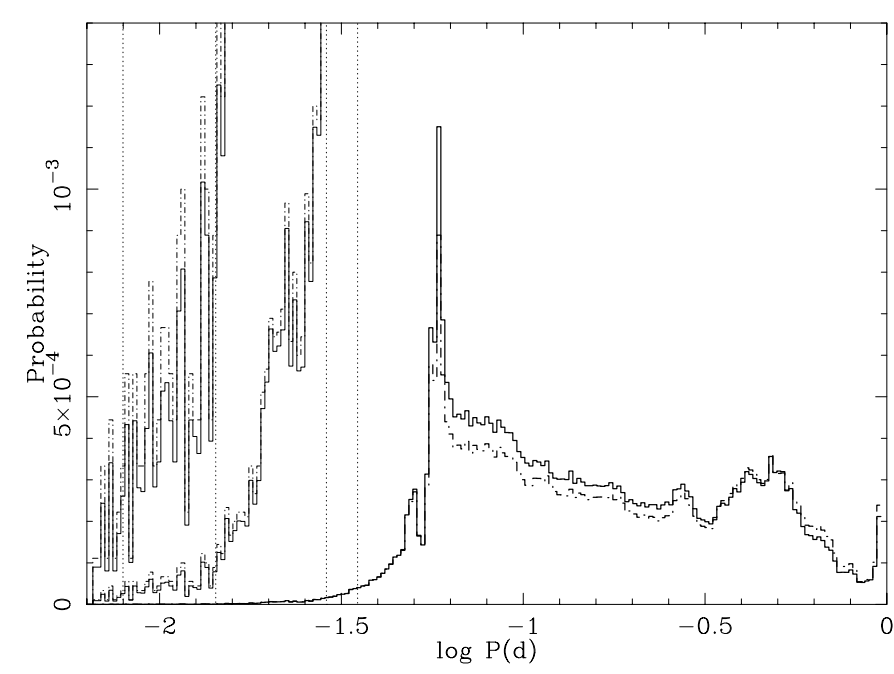

Fig. 10. Probability distribution of the orbital periods for all models with $Z=0.02$. The characteristics of this plot are the same as in Fig. 6 .

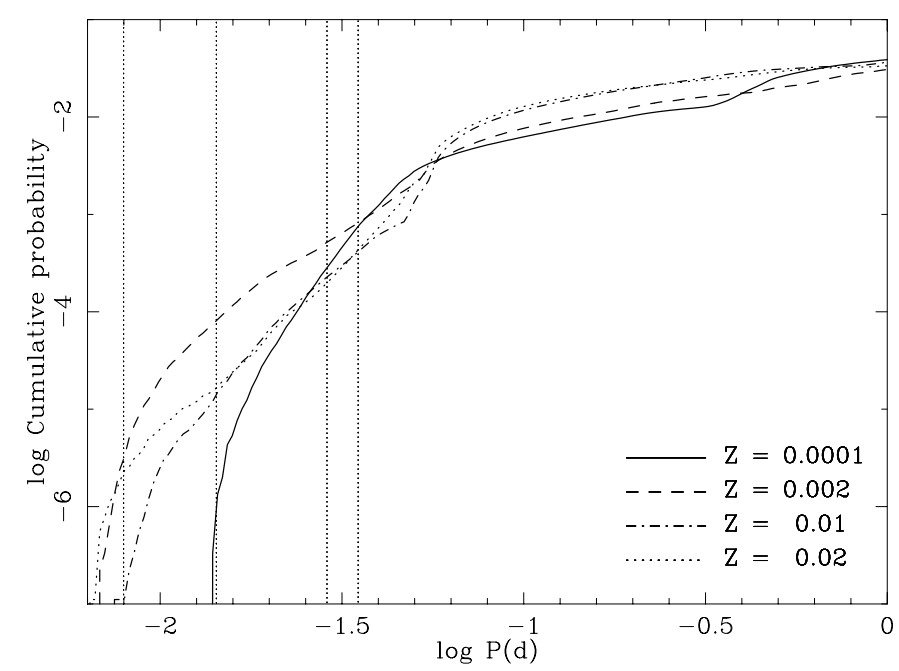

Fig. 11. Cumulative plot for the distribution of the orbital periods for all models and all four metallicities. The different line styles represent the different metallicities as indicated in the lower right of the plot. The height of the lines shows the logarithm of the fraction of all probed systems that have an orbital period equal to or lower than the period on the horizontal axis. For all lines, a flat initial mass distribution is used. The dotted vertical lines show the observed orbital periods mentioned in Fig. 1.

distribution. For the ultra-short periods, there is very little difference between the two weighing methods, and we can again conclude that the exact initial mass distribution is not important for our results.

We also see that the lowest possible orbital period for an $\mathrm{X}$-ray binary with $Z=0.0001$ within the Hubble time is about a factor two smaller than for the other metallicities. This is partly due to the fact that ultra-compact binaries are less likely to be formed for this low metallicity because the initial period must be chosen more precisely. However, we find no minimum periods less than $16.0 \mathrm{~min}$ for this metallicity. This has probably to do with the fact that these stars are hotter and thus have a weaker magnetic field.
In a sample of $10^{7}$ binaries with $Z=0.0001$, we expect no converging systems with mass transfer and an orbital period of $11.4 \mathrm{~min}$, and around 5 with a $20.6 \mathrm{~min}$ period (Fig. 8). For $Z=0.002$ and $Z=0.02$, these numbers are 7 systems with an $11.4 \mathrm{~min}$ period and 60 with a $20.6 \mathrm{~min}$ period and 4 systems with an $11.4 \mathrm{~min}$ period and 10 with a $20.6 \mathrm{~min}$ period respectively.

Figure 11 shows clearly that there is some difference between the period distributions for the different metallicities, the largest difference being the higher period cut-off for the lowest orbital periods for $Z=0.0001$. The largest differences for the three higher metallicities are found around $11 \mathrm{~min}$, (a bit more than an order of magnitude between $Z=0.01$ and the other two metallicities) and around $20 \mathrm{~min}$ (less than an order of magnitude between $Z=0.002$ and the others). Note that the line for $Z=0.01$ predicts for each system with an orbital period of 11 min about 100 systems with $P_{\text {orb }} \lesssim 20$ min.

\section{Discussion}

\subsection{The importance of converging evolution for the formation of ultra-compact binaries}

To understand why the fraction of ultra-compact binaries with decreasing orbital period in our computations is so small, we note that there are three main factors contributing to this. First, only a limited range of initial orbital periods leads to strongly converging orbital evolution within the Hubble time, as listed in Table 3. This range of periods varies strongly with donor mass: for $Z=0.01$ and for 1.0 and $1.1 M_{\odot}$ the width is about $0.1 \mathrm{~d}$; but for 1.2 and $1.3 M_{\odot}$ it is only $0.003 \mathrm{~d}$. This corresponds to $\sim 5 \%$ and $\sim 0.2 \%$, respectively, of the range that we consider. The reason for this rapid decrease is that the nuclear evolution time scale of the star increases much more rapidly with mass than the time scale of magnetic braking. Thus, at higher stellar mass magnetic capture can only occur for smaller initial orbital periods. Second, for each initial orbital period within the range of converging systems, only a very short time is spent at ultra-short periods while converging. Thus, the $1.1 M_{\odot}$ system with initial period of $0.85 \mathrm{~d}$ reaches the $20 \mathrm{~min}$ period after $11.163 \mathrm{Gyr}$ and the $11 \mathrm{~min}$ period after $11.167 \mathrm{Gyr}$. If we allow a range of ages of $3 \mathrm{Gyr}$, then only $0.1 \%$ of these systems will have an orbital period less than $20 \mathrm{~min}$ and a negative period derivative. If we allow also positive period derivatives, the fraction of ultra-compact binaries is somewhat higher: as can be seen in Fig. 2 the evolution towards longer period is comparably rapid as the evolution towards shorter period close to the minimum period. Third, as already mentioned, the range of initial periods leading to converging systems is very small for donors with $M \geq 1.2 M_{\odot}$; hence only donors in a narrow range of initial masses contribute to ultra-short period systems. The combination of these three factors explains why so few ultra-short period systems are produced, as already surmised by Tutukov et al. (1987).

In our computations above we have assumed an initial period distribution in the range $0.5 \mathrm{~d} \lesssim P_{\mathrm{b}} \lesssim 3 \mathrm{~d}$. In the galactic disk, the actual period range extends to much longer periods, and accordingly our estimates of the fraction of X-ray 
Table 3. Comparison between the orbital periods that lead to periods less than $30 \mathrm{~min}$ within a Hubble time and orbital periods that result from tidal capture with a $1.4 M_{\odot}$ neutron star, for different secondary masses and $Z=0.01$. Column 1: initial secondary mass, Cols. 2 and 3: initial period range that leads to ultra-short periods, Cols. 4 and 5: RLOF-period period range that leads to ultra-short periods, Cols. 6 and 7: ZAMS and TAMS radii, Cols. 8 and 9: orbital periods for a circularised binary with capture distances of $1 \times R_{\text {zams }}$ and $3 \times R_{\text {tams }}$. Masses are in $M_{\odot}$, radii in $R_{\odot}$ and periods in days.

\begin{tabular}{lllllllll}
\hline \hline$M_{\mathrm{i}}$ & $P_{\mathrm{i}, 1}$ & $P_{\mathrm{i}, 2}$ & $P_{\text {rlof }, 1}$ & $P_{\text {rlof }, 2}$ & $R_{\text {zams }}$ & $R_{\text {tams }}$ & $P_{\text {zams }}$ & $\mathrm{P}_{\text {tams }}$ \\
\hline 1.0 & 1.477 & 1.589 & 0.638 & 0.715 & 0.92 & 1.73 & 0.19 & 2.50 \\
1.1 & 0.767 & 0.856 & 0.640 & 0.740 & 1.05 & 1.51 & 0.22 & 2.00 \\
1.2 & 0.753 & 0.756 & 0.686 & 0.689 & 1.18 & 1.71 & 0.26 & 2.37 \\
1.3 & 0.753 & 0.756 & 0.704 & 0.707 & 1.27 & 2.00 & 0.29 & 2.94 \\
1.4 & 0.753 & 0.758 & 0.714 & 0.719 & 1.31 & 2.37 & 0.29 & 3.71 \\
1.5 & 0.752 & 0.763 & 0.717 & 0.728 & 1.33 & 2.65 & 0.29 & 4.32 \\
\hline
\end{tabular}

binaries that is observed at ultra-short periods are upper bounds, for systems evolved along the scenario that we compute. This is in agreement with the absence of large numbers of $\mathrm{X}$-ray binaries with periods much less than $40 \mathrm{~min}$, in the galactic disk. If fact only one such system has recently been discovered; it may well have formed through a different mechanism, e.g. via a double spiral-in at the end of which a white dwarf becomes the donor of a neutron star (Savonije et al. 1986).

In globular clusters the binary period distribution is expected to be different from that in the galactic disk: the widest primordial binaries are dissolved and close binaries are produced in close stellar encounters. If the neutron star is exchanged into a primordial binary in a neutron-star/binary encounter, the period after the encounter scales with the preencounter binary period; in general the orbit after exchange will be similar in size (Sigurdsson \& Phinney 1993). However, the range of periods is still expected to be wider than the range that we have considered in our computations, which therefore give an upper bound to the fraction of ultra-compact binaries. If the neutron star is captured tidally, the orbital period after capture tends to be short. The exact description of tidal capture is highly uncertain, and we will discuss the simplest description to provide a reference frame. In this description, the neutron star captures a main-sequence star if its closest approach $d$ is within three times the radius $R$ of that star, i.e. $d \leq 3 R$ (Fabian et al. 1975). The capture rate is linear in $d$; thus one third of the captures is a direct hit, which completely destroys the mainsequence star. Capture may lead to a binary if $R \lesssim d \leq 3 R$. The lower bound may in fact be higher, since too close a capture still does serious damage to the star (Ray et al. 1987). The orbit immediately after capture is highly eccentric, and after it circularises its semi-major axis is twice the capture distance: $a_{\mathrm{c}} \simeq 2 d$. Hence orbits formed by tidal capture have a semi-major axis (after circularisation) $2 R \lesssim a_{\mathrm{c}} \leq 6 R$, or with Kepler's law:

$$
\begin{aligned}
0.23 \mathrm{~d}\left(\frac{R}{R_{\odot}}\right)^{3 / 2}\left(\frac{M_{\odot}}{M+m}\right)^{1 / 2} & \lesssim P_{\mathrm{b}} \\
& \leq 1.20 \mathrm{~d}\left(\frac{R}{R_{\odot}}\right)^{3 / 2}\left(\frac{M_{\odot}}{M+m}\right)^{1 / 2}
\end{aligned}
$$

Immediately after the capture, the main-sequence star is highly perturbed, but after a thermal timescale it may settle on its equilibrium radius, and continue its evolution. The range of orbital periods depends on the radius that the star has when it is captured. In general, the period range is bounded below by the period found by entering twice the zero-age main-sequence radius into Eq. (5) and above by entering six times the terminal-age main-sequence radius (because a star evolved beyond this point does not evolve towards shorter periods). In Table 3 we list the period ranges expected in this simplest description of capture. Unless the central density of the globular cluster evolves dramatically, the probability of capture is approximately flat in time. The period after capture close to the zero-age main sequence should be compared to the initial binary period in our computations; the period after capture close to terminal-age main sequence should be compared to the period of a system close to filling its Roche lobe. In either case, we see that capture leads to a period distribution which covers an appreciable fraction of the period distribution that we cover in our computations. This means that our conclusion that only an exceedingly small fraction of all binaries with a neutron star evolve towards periods less than $30 \mathrm{~min}$ holds also for tidally captured binaries.

We have taken the simplest description of tidal capture. From the above argument it is clear that changing the assumptions made about tidal capture is unlikely to change our conclusion, that evolution from magnetically driven converging evolution does not contribute significantly to the population of ultra-compact binaries. Even if tidal capture would miraculously focus the resulting orbits into the narrow range required for converging evolution, the fact would remain that each systems spends only a small fraction of its time converging from $20 \mathrm{~min}$ to $11 \mathrm{~min}$.

If the binary in NGC 6624 were the only ultra-short-period binary in a globular cluster, one could accept an evolutionary scenario with low probability. It is thus worthy of note that our statistical argument depends critically on the observation that the $20.6 \mathrm{~min}$ ( or $13.2 \mathrm{~min}$ ) period of the binary in NGC 6712 is real. So far, this period has been measured only once in a single HST data set, and an independent new measurement is very desirable, to exclude definitely that the first measurement of a significant periodicity is a statistical fluke.

\subsection{Comparison to Pylyser \& Savonije}

The question arises why Pylyser \& Savonije (1988) and Pylyser \& Savonije (1989), hereafter PS1 and PS2, did not find ultracompact systems in their study. We tried to reproduce their models with a $1.0 M_{\odot}$ compact primary and a $1.5 M_{\odot}$ secondary (models A25-I25 in PS1 and A25-Z25 in PS2) because these are best documented and they find the lowest minimum period here (38 min for A25 in PS2). We calculated models with the same initial masses, mixing length $\left(l / H_{\mathrm{p}}=1.5\right)$, metallicity $(Z=0.02)$ and without overshooting. Figure 12 compares their results to our calculations as the minimum period $\left(P_{\min }\right)$ as a function of the period where Roche-lobe overflow starts $\left(P_{\text {rlof }}\right)$.

We find the bifurcation period at much larger $P_{\text {rlof }}$, which is due to the fact that our stars rotate (about $25 \%$ of the difference, 


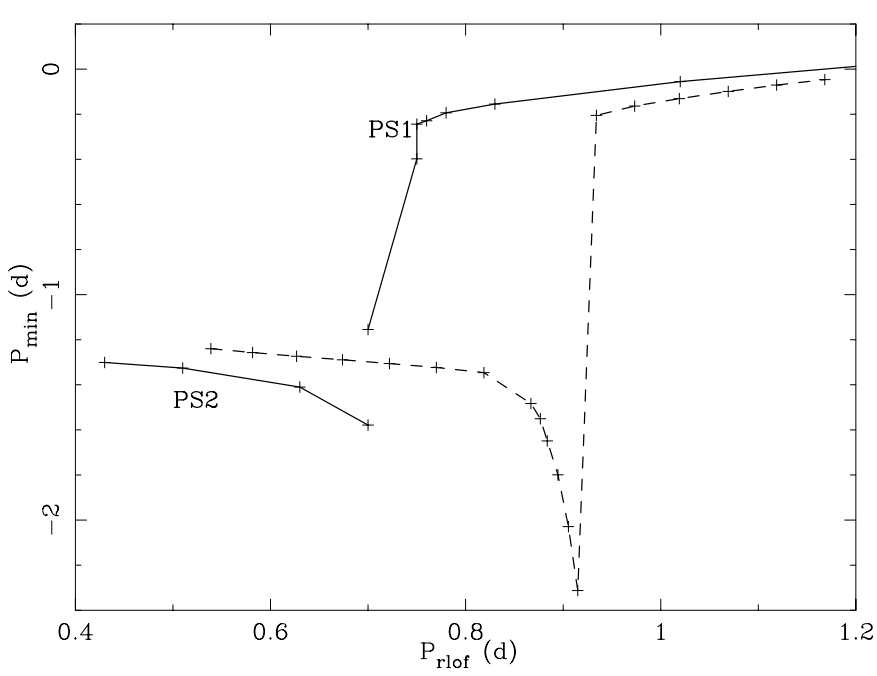

Fig. 12. Comparison of our models (dashed line) to the models A25-I25 of PS1 and A25-Z25 of PS2 (solid lines). See the text for details.

according to test calculations we have done) and increased opacities. Both effects increase the radii of our model stars, so that they must be placed in a larger orbit to fill their Roche lobe at the same stage of evolution. It seems that shifting the two solid lines of PS1 and PS2 horizontally can approximately compensate for this, but the lines must be shifted over different amounts. Hence, a gap arises between what at first sight appears to be a continuous $P_{\text {rlof }}$-range from PS1 and PS2. The fact that they find the minimum period at the limit of each range, and the fact that these two points are both at $P_{\text {rlof }}=0.70 \mathrm{~d}$, but give very different minimum periods ( $100 \mathrm{~min}$ and $38 \mathrm{~min}$ for PS1 and PS2 respectively), are supporting the evidence for the existence of this gap. The cause for the gap seems to be clear; in PS2 an accretion induced collapse (AIC) occurs when the primary reaches $1.44 M_{\odot}$, whereas in PS1 no such event happens. The AIC decreases the mass of the compact object and increases the orbital period so that the further evolution can no longer be compared to that of systems without an AIC.

In our more complete series of models, shown in Fig. 12, the lowest minimum period we find is $7.0 \mathrm{~min}$, and is reached after $12.4 \mathrm{Gyr}$.

\subsection{Comparison to Podsiadlowski et al.}

We chose the parameters of our models as similar as possible to those of Podsiadlowski et al. (2002) (see Sect. 2), to see if we could reproduce their results for a $1.4 M_{\odot}$ neutron star and a $1.0 M_{\odot}$ secondary. Indeed, the results of our calculations are qualitatively very similar to their findings in their Fig. 16 and their statement that binaries with an orbital period of 5 min can be achieved without a spiral-in, although we need slightly larger initial periods to get to the same minimum period. Podsiadlowski et al. (2002) display their results as a function of time since Roche-lobe overflow started, and because of this we cannot ascertain the total age of the binary at the minimum period. The red and blue model in their Fig. 16 reach minimum periods of about 9 and $7 \mathrm{~min}$, at approximately 4.5 and 5.5 Gyr after the beginning of RLOF. We find very similar results, and in addition we find the total ages of these systems: 14 and 17 Gyr respectively. We find that it takes $13.4 \mathrm{Gyr}$ to reach an orbital period of $11.4 \mathrm{~min}$, the shortest period observed for an X-ray binary, and more than 35 Gyr to shrink the orbit to $5 \mathrm{~min}$. We conclude that it is not possible to create systems with orbital periods less than 10 min this way, within a Hubble time.

Podsiadlowski et al. (2002) find that there is a rather large range of initial orbital periods $(13-17.7 \mathrm{~h})$ that lead to a minimum period that is less than $30 \mathrm{~min}$. We find for the same condition a $P_{\text {rlof }}$ of $15.3-17.2 \mathrm{~h}$, which is considerably smaller. This is firstly because our model stars have a slightly larger radius. Part of the explanation of the increased radius is given by the rotation of the star, although this can only account for $20 \%$ of the difference in the $P_{\text {rlof }}$-range, and by the different helium abundance (Podsiadlowski et al. (2002) use $Y=0.27$, we have $Y=0.26$ ), which explains $10 \%$. The larger radius shifts the whole $P_{\text {rlof }}$-range to larger orbital period. Secondly, we limit our range to systems that reach their minimum period before the Hubble time, so that it is cut off above a certain $P_{\text {rlof }}$.

What Podsiadlowski et al. (2002) call the initial period is the period at which RLOF initiates, and which we call $P_{\text {rlof }}$. In the time before RLOF began, the magnetic braking may have played a role in shrinking the pre-RLOF orbit of the systems as listed in Table 3.

\subsection{Comparison with observations and other models}

The main result from our computations is that, in a population where all X-ray binaries evolve from close detached binaries of a main-sequence star and a neutron star, systems with orbital periods less than 30-40 min and with decreasing orbital periods are very rare. If we accept that the orbital period of the X-ray source in NGC 6624 is decreasing intrinsically (and not just observationally due to gravitational acceleration), we must accept that it is a statistical fluke, or look for a different origin.

In this respect it would be important to know more about the orbital periods and their derivatives of other X-ray sources in globular clusters. A very short orbital period is detected for just one other bright X-ray source, in NGC 6712, as a regular variation of $0.044(7) \mathrm{mag}$ in one series of $53 \mathrm{F300W}$ (wide $U$ ) filter HST observations with WFPC2 in 1995; aliasing allows two solutions at 13.2 or $20.6 \mathrm{~min}$ (Homer et al. 1996). Homer et al. (1996) opt for the longer period, on the basis of the low $\mathrm{X}$-ray luminosity that reflects a low mass-transfer rate and a model in which the donor to the neutron star is a white dwarf (Verbunt 1987). We note that the same choice for the longer period would follow for the magnetic-capture model. The period derivative of the source in NGC 6712 is not known. The argument that as many as half of the bright X-ray sources in globular clusters have ultra-short periods is based on the similarity of various properties of those X-ray sources with the properties of the X-ray sources in NGC 6624 and NGC 6712. This argument is correct only if the X-ray source in NGC 6712 indeed has an ultra-short period. It is therefore important that this period is 
confirmed; which will also settle between the aliases of 13.2 and $20.6 \mathrm{~min}$.

Measurement of the period derivative will be very difficult. It is therefore of interest to know how many ultra-compact binaries one would expect irrespective of their period derivative, in the magnetic capture model. Alas, our computations stop a short time after the minimum period, so that we do not have an accurate estimate of the time spent at positive period derivative. Nonetheless, inspection of our results as reflected in Fig. 1 shows that the evolution away from the minimum period is only slightly slower than the evolution towards it. Thus, the number of systems expected at the shortest period range of between 10-30 min would only be a factor few higher than the number in the same period range with decreasing period only. This implies that the presence of even two systems with periods less than $30 \mathrm{~min}$ among 13 globular cluster systems excludes the magnetic-capture scenario as the dominant formation process. The conclusion is true a fortiori if more such systems are discovered.

A donor in an ultra-compact system can also be a heliumburning star. To bring such a small star into contact, a spiralin must have occurred (Savonije et al. 1986). The progenitor of such a helium-burning star would be more massive than the main-sequence star found in globular clusters, and Verbunt (1987) argued that this excludes such donors for sources in globular clusters. However, more massive stars can be made in direct collisions: if such a more massive star ends up in a binary with a neutron star, further evolution can lead to a heliumburning donor in an ultra-compact system. This scenario may gain in importance if tidal capture is indeed less efficient, as indicated by a high fraction of systems with ultra-short periods. It allows negative derivatives of the orbital period.

Since the measurement of the intrinsic derivative of the orbital period is so difficult, it is useful to look for other observational properties that can discriminate between the different origins of an ultra-compact binary. With this in mind, we refer to Table 2 where some properties of ultra-short-period systems are listed that follow for the magnetic-capture model, in particular the mass-transfer rate at various periods, and the abundances of the more important elements. A pure white-dwarf donor, whittled down to a mass less than $0.1 M_{\odot}$, would have no hydrogen if it was a helium white dwarf; and no hydrogen and no helium if it was a carbon-oxygen white dwarf. Therefore, if hydrogen is discovered in the spectrum of an ultra-compact $\mathrm{X}$-ray binary, this indicates evolution through magnetic capture and the orbital period must still be decreasing. Close to the minimum period the hydrogen abundance at the surface goes to zero and thus is no longer discriminant between models.

Acknowledgements. We thank P. P. Eggleton for making available to us the latest version of his binary evolution code. We also thank F. Rasio for his suggestions to improve this article.

\section{References}

Alexander, D. R., \& Ferguson, J. W. 1994, ApJ, 437, 879

Chou, Y., \& Grindlay, J. E. 2001, ApJ, 563, 934

Davies, M. B., \& Hansen, B. M. S. 1998, MNRAS, 301, 15

Deutsch, E. W., Anderson, S. F., Margon, B., \& Downes, R. A. 1996, ApJ, 472, L97+

Eggleton, P. P. 1971, MNRAS, 151, 351

Eggleton, P. P. 1972, MNRAS, 156, 361

Eggleton, P. P., \& Kiseleva-Eggleton, L. 2002, ApJ, 575, 461

Fabian, A. C., Pringle, J. E., \& Rees, M. J. 1975, MNRAS, 172, 15

Heinke, C. O., Edmonds, P. D., \& Grindlay, J. E. 2001, ApJ, 562, 363

Homer, L., Charles, P. A., Naylor, T., et al. 1996, MNRAS, 282, L37

Hut, P. 1981, A\&A, 99, 126

Iglesias, C. A., Rogers, F. J., \& Wilson, B. G. 1992, ApJ, 397, 717

Kuulkers, E., den Hartog, P. R., in't Zand, J. J. M., et al. 2003, A\&A, 399,663

Paczynski, B., \& Sienkiewicz, R. 1981, ApJ, 248, L27

Peters, P. 1984, Phys. Rev., 136, 1224

Podsiadlowski, P., Rappaport, S., \& Pfahl, E. D. 2002, ApJ, 565, 1107

Pols, O. R., Tout, C. A., Eggleton, P. P., \& Han, Z. 1995, MNRAS, 274, 964

Pols, O. R., Tout, C. A., Schröder, K., Eggleton, P. P., \& Manners, J. 1997, MNRAS, 289, 869

Pylyser, E., \& Savonije, G. J. 1988, A\&A, 191, 57

Pylyser, E. H. P., \& Savonije, G. J. 1989, A\&A, 208, 52

Rappaport, S., Joss, P. C., \& Verbunt, F. 1983, ApJ, 275, 713

Rasio, F. A., Pfahl, E. D., \& Rappaport, S. 2000, ApJ, 532, L47

Rasio, F. A., \& Shapiro, S. L. 1991, ApJ, 377, 559

Ray, A., Kembhavi, A. K., \& Antia, H. M. 1987, A\&A, 184, 164

Savonije, G. J., de Kool, M., \& van den Heuvel, E. P. J. 1986, A\&A, 155,51

Schröder, K., Pols, O. R., \& Eggleton, P. P. 1997, MNRAS, 285, 696

Sidoli, L., Parmar, A., Oosterbroek, T., et al. 2001, A\&A, 368, 451

Sigurdsson, S., \& Phinney, E. S. 1993, ApJ, 415, 631

Stella, L., Priedhorsky, W., \& White, N. E. 1987, ApJ, 312, L17

Tutukov, A. V., Fedorova, A. V., Ergma, E. V., \& Yungel'Son, L. R. 1985, Pis ma Astron. Zh., 11, 123

Tutukov, A. V., Fedorova, A. V., Ergma, E. V., \& Yungelson, L. R. 1987, Soviet Astronomy Letters, 13, 328

van der Klis, M., Hasinger, G., Verbunt, F., et al. 1993, A\&A, 279, L21

van Paradijs, J., \& McClintock, J. E. 1994, A\&A, 290, 133

Verbunt, F. 1987, ApJ, 312, L23

Verbunt, F., \& Lewin, W. 2004, in Compact stellar X-ray sources, ed. W. Lewin, \& M. van der Klis (Cambridge: Cambridge U.P.)

Wang, Z., \& Chakrabarty, D. 2004, ApJ, submitted

[arXiv: astro-ph/0406465]

Webbink, R. F., Rappaport, S., \& Savonije, G. J. 1983, ApJ, 270, 678

Yungelson, L. R., Nelemans, G., \& van den Heuvel, E. P. J. 2002, A\&A, 388, 546 